\title{
Premenstrual syndrome: etiology, diagnosis and treatment. A mini literature review
}

\author{
Kelechi Martins Nworie
}

Pharmaceutical Outcomes Research Group, Faculty of Pharmacy, University of Nigeria, Nsukka, Nigeria

\begin{abstract}
Premenstrual syndrome (PMS) is characterized by a combination of emotional, physical, psychological, and mood disturbances that occur after a woman's ovulation, typically ending with the onset of her menstrual flow. Premenstrual dysphoric disorder (PMDD) is a severe condition of PMS which can be very disabling, as it affects occupational activities and personal relationships. Globally, PMS occurs in 30-40\% of women of reproductive age, with 3-8\% of this population being affected by PMDD. Despite several studies, the etiology of PMS still remains unclear; however, most theories suggest that PMS has increased sensitivity to normal hormonal changes as well as neurotransmitter abnormalities. Treatment of PMS often requires pharmacological interventions. Serotonergic antidepressants are the mainstay for improving both physical and mood symptoms. Oral contraceptives are also effective for relieving physical symptoms. Other non-pharmacological interventions include lifestyle modification and cognitive behavioral therapy.

Key words: premenstrual syndrome, premenstrual dysphoric disorder, neurotransmitter, oral contraceptive, serotonergic antidepressant, exercise.
\end{abstract}

Kelechi Martins Nworie, Department of Clinical Pharmacy and Pharmacy Administration, Pharmaceutical Outcomes Research Group, Faculty of Pharmacy, University of Nigeria, Nsukka, Nigeria, phone: +234(0)8063728781, e-mail: nworiekelechimartins@gmail.com 


\section{Introduction}

Premenstrual disorders consist of psychiatric or somatic symptoms that develop within the luteal phase of the menstrual cycle, affect the patient's normal daily functioning, and resolve shortly after menstruation [1]. The onset of the luteal phase is after ovulation and terminates at the beginning of menstruation. Despite many controversies, authorities in the fields of psychiatry and gynecology have developed distinct diagnoses for premenstrual syndrome (PMS) and premenstrual dysphoric disorder (PMDD) [1]. One of these authorities, the American College of Obstetricians and Gynecologists (ACOG), made a significant contribution towards understanding PMS [2]. The ACOG includes psychiatric and physical symptoms in defining PMS. The American Psychiatry Association (APA) focuses mainly on psychiatric symptoms as criteria for diagnosis of PMDD [3]. These symptoms predominate between menarche and menopause. The consequences of the disease can be severe in some individuals; higher rates of work absences or academic absences, higher medical expenses, and lower health-related quality of life are often observed in women with premenstrual syndrome [4].

\section{Epidemiology}

During the luteal phase of the menstrual cycle, approximately $80 \%$ of women report at least one physical and psychiatric symptom; however, daily activity is not impaired in most of them [5]. Persistence of symptoms tends to fluctuate in women, but its prevalence is not associated with age, educational attainment or wealth status [6]. One study reported that approximately $36 \%$ of women had PMS, and a follow-up observation showed that these women continued to meet diagnostic criteria for PMS after 1 year [6]. Weight gain and indulgence in stressful events were significant predictors of PMS $[6,7]$. The severity of premenstrual syndrome in women varies [4]. The severe and extreme form of PMS called premenstrual dysphoric disorder (PMDD) occurs in 3-8\% of women [8]. Studies have shown that approximately $30-50 \%$ of women experience a mild to moderate form of PMS for several days [8].

\section{Etiology}

The key challenge of premenstrual syndrome is poor understanding of its etiology [6,9]. Body tissues of a woman becomes sensitive to changes in hormone levels throughout the menstrual cycle. Changes in levels of hormones (estrogen and progesterone) may influence chemical in the brain, especially serotonin, which affects mood [10]. Studies suggest that these symptoms are triggered by rising and falling of estrogen and progesterone levels [11, 12]. A possible explanation for this is that post-menopausal women previously diagnosed with PMS have recurrence of symptoms when undergoing progesterone therapy [12]. Furthermore, gonadotropin-releasing hormone analogs which suppress estrogen significantly reduce PMS symptoms $[13,14]$. The cyclic effect of estrogen and progesterone on serotonin, $\gamma$-aminobutyric acid, and dopamine systems may be the cause of changes in mood $[11,13]$. These mechanisms can alter the renin-angiotensin-aldosterone (RAS) system, which could possibly explain some symptoms such as bloating, cramps, swelling and weight gain that occur during PMS [11]. Estrogen and progesterone levels in women with or without the condition of PMS are the same [6]. However, why some women develop PMS and others do not is unclear, as studies have shown that women diagnosed with PMS do not have a higher level of estrogen and progesterone than other women [6]. The most likely explanation for this, based on several studies, is that women who develop PMS are more sensitive to normal changes in hormone levels during the menstrual cycle [10-12]. Although no genes have been identified indicating hereditary nature of PMS, some studies suggest there could be a possible genetic component to premenstrual disorders [15]. The most common symptoms of PMS are fatigue, bloating, irritability, depression, and anxiety [16]. Other symptoms include sadness, hopelessness, tension, mood swings, difficulty concentrating, appetite changes, insomnia, swelling, and headaches. Premenstrual syndrome may lead to capacity loss in women, thereby causing psychological effects such as anxiety, depression, and suicide attempts, which results in reduced health-related quality of life [1, 3-5].

\section{Diagnosis}

The ACOG has defined PMS as a condition in which a woman experiences at least one affective symptom and one somatic symptom that cause dysfunction in social, academic, or work performance [2]. The peculiarity of these symptoms lies in their cyclical nature, usually beginning at the luteal phase and immediately after menstruation. It was noted that the cyclical luteal timing is important for assessing PMS; symptoms must occur after ovulation and resolve immediately after the onset of menstruation [17]. Certain health conditions including migraine, depression, anxiety disorders, anemia, fibromyalgia, endometriosis, hypothyroidism or hyperthyroidism have symptoms comparable to those of PMS, and may suggest an underlying illness $[18,19]$. It is essential to distinguish between underlying illness and true PMS because they have quite different treatment protocols and should also be considered during diagnosis. A careful medical history should be able to distinguish between these conditions. Women with underlying depression may feel better during or after menses, but do not resolve completely, unlike symptoms in PMS, which resolve completely at onset of menses. Some women may think they have PMS while they actually have depression or anxiety disorder.

There is no single method for diagnosis of PMS [3]. Laboratory diagnostic testing is not necessary but can be used complementarily. For instance, blood count can be recommended to screen for anemia, and thyroid function to detect hypothyroidism and hyperthyroidism. Prospective questionnaires such the Daily Record of Severity of Problems (DRSP), Calendar of Premenstrual Experiences (COPE) 
among others are the most valid and reliable tools for diagnosis of PMS [2, 3, 20]. However, these methods require immense time and effort since patients have to report their symptoms daily for at least two menstrual cycles.

\section{Treatments}

Pharmacological intervention is usually the best approach for treatment of women with PMS. Since premenstrual symptoms are a normal body phenomenon, treatment of PMS mainly centers on alleviating physical and psychiatric symptoms. Medications used mainly affect the hormonal activity through repression of ovulation, while others affect neurotransmitter levels in the brain [21]. These neurotransmitters may include norepinephrine, dopamine, and serotonin. For instance, the selective serotonin reuptake inhibitors (SSRIs) are used as primary treatment of PMS in the United States. The SSRIs are basically psychiatric medications, but they also improve physical symptoms when used to treat PMS in patients [21]. Therapy for PMS should be strictly modified based on patient tolerance.

\section{Pharmacological treatments \\ Serotonergic antidepressants}

The first-line medications recommended for treatment of PMS are the SSRIs. They are highly effective treatment for highly psychiatric symptoms of PMS. These SSRIs include fluoxetine (Prozac and Sarafem), sertraline (Zoloft), paroxetine (Paxil), citalopram (Celexa) and escitalopram (Lexapro). Multiple studies have now confirmed that SSRIs reduce the psychiatric symptoms, as well as some physical symptoms of PMS [21]. A review analysis of randomized controlled clinical trials showed that the five SSRIs relieved symptoms of PMS compared to placebo [21]. Each SSRI had significant benefits on patient-reported symptoms when taken during the luteal phase. In a fluoxetine trial, doses of 20-60 mg daily resulted in a 53\% improvement in PMS symptoms [22]. The multicenter randomized clinical trial of sertraline at doses of 50-150 mg reported a 62\% improvement in PMS symptoms [23]. Several trials have also demonstrated the efficacy of other SSRIs including paroxetine, fluvoxamine, and citalopram [24-26]. In the citalopram trial, luteal administration alone was found to be more effective than continuous administration [26]. It may not be necessary to take the SSRI medication on a daily basis; hence, taking the SSRIs just a few days prior to menses may be enough. Common adverse effects of SSRIs include gastrointestinal tract disturbances, fatigue and sleep problems [21]. Some women also have sexual dysfunction as side effects [21]. This might manifest as difficulty having an orgasm. In such a situation, titrating to a lower dose or trying alternative medication is recommended. All SSRIs are effective for treating psychiatric symptoms of PMS, and medication should be tailored to the patient's tolerability [21]. The SSRIs for treatment of PMS are initiated at doses lower than the standard doses recommended for treating depression. Standard starting doses of SSRIs for PMS are fluoxetine, $10 \mathrm{mg}$, sertraline, $25 \mathrm{mg}$, and paroxetine, 5-10 mg.

\section{Serotonin-norepinephrine reuptake inhibitors (SNRIs)}

Serotonin-norepinephrine reuptake inhibitors such as venlafaxine have been administered to treat PMS in women with predominant psychological symptoms [27]. A randomized controlled trial involving 164 women compared venlafaxine with placebo for symptom relief in PMS [27]. Venlafaxine was significantly more effective than placebo in relieving symptoms of PMS [27]. Sixty percent of venlafaxine versus $35 \%$ of placebo subjects improved more than $50 \%$. Adverse effects include nausea, insomnia, and dizziness [27].

\section{Oral contraceptives}

Despite insufficient evidence for efficacy, oral contraceptives are often prescribed for PMS. Although it is widely established that oral contraceptives will improve dysmenorrhea, their effectiveness for psychiatric symptoms is not fully recognized. However, some studies have demonstrated that some women with PMS obtain relief for psychiatric and physical symptoms when treated with oral contraceptives [28, 29]. A double-blind, placebo-controlled study of an oral contraceptive found improvement in bloating and breast pain but no significant improvement in mood symptoms [28]. Another study which analyzed four randomized controlled trials that compared continuous administration of oral contraceptive ( $90 \mu \mathrm{g}$ levonorgestrel/20 $\mu \mathrm{g}$ ethinyl estradiol) and placebo in women with premenstrual disorders found improvement in depression and physical symptoms [29]. Another randomized, placebo-controlled study in women with premenstrual disorder found that the oral contraceptive Yasmin (containing low-dose ethinylestradiol combined with a progesterone and drospirenone) had consistently significant reduction of 22 premenstrual symptoms assessed using the Calendar of Premenstrual Experiences [30]. The effect of oral contraceptives on symptoms of PMS are still in progress. The use of natural progesterone administered in a wide range of doses and routes was the centerpiece of therapy for PMS a few decades ago, despite absence of supportive evidence from clinical trials. However, several randomized, placebo-controlled trials have investigated the use of natural progesterone for PMS symptoms and the results were negative $[31,32]$.

\section{Gonadotropin-releasing hormone (GnRH) agonists}

Gonadotropin-releasing hormone agonists such as leuprolide acetate or goserelin acetate are a type of medication that modulates pituitary GnRH receptors, with the subsequent induction of hypoestrogenic amenorrheic condition [33]. Gonadotropin-releasing hormone agonists cause temporal cessation of synthesis of estrogen and progesterone by the ovaries, which is beneficial for physical symptoms of PMS. Several studies have illustrated the efficacy of GnRH analogs [34]. However, a concern over GnRH analogs is the impact of prolonged extremely low estrogen levels which mimic symptoms of menopause such as severe hot flashes and accelerated bone loss [34]. A clinical trial examined the use of low dose estrogen and progesterone in hormone replacement therapy to stop hot flashes and bone loss, in addition to GnRH agonists for treating PMS [35]. This trials 
demonstrated the efficacy for PMS. GnRH agonist therapies are very effective. However, due to complications and cost, their use in PMS is limited. They are only recommended for use if other treatments fail.

\section{Psychotherapeutic agents}

Some psychotherapeutic agents which can be classified as anxiolytics and antidepressants have exhibited a beneficial impact for PMS in certain clinical trials. These drugs include alprazolam and buspirone. Alprazolam is a benzodiazepine that relieves symptoms of PMS at doses of $0.25 \mathrm{mg}$ orally three times daily $[32,36]$, but care is taken during administration to avoid dependence associated with benzodiazepines [36]. Buspirone is a non-benzodiazepine that has demonstrated efficacy in placebo-controlled trials with doses of 25-60 mg daily [37].

\section{Calcium supplements}

Administration of calcium supplements has been demonstrated as relieving PMS symptoms. Although the precise mechanism of action is unspecified, consistent cyclical changes in body calcium levels were observed in women with PMS and mood disorder [38]. A small randomized controlled trial of female students with PMS demonstrated the efficacy of supplemental calcium compared to placebo; women who received $500 \mathrm{mg}$ of calcium carbonate supplement twice daily for 3 months showed a $50 \%$ reduction in depression, appetite change, and fatigue [39]. A similar trial of 400 women in the US who supplemented with $1200 \mathrm{mg}$ daily of calcium carbonate produced the same result [38].

\section{Vitamin and mineral supplements}

Vitamin and minerals may possess little beneficial impact, especially for women with mild PMS. However, the precise association of vitamin B6 with PMS in studies has been thwarted by mixed results, and this has clearly hampered a firm conclusion about the benefit of vitamins $B_{6}$ in PMS. Vitamin $B_{6}$ at doses of 60-100 mg daily has been recommended as treatment for some psychiatric symptoms of PMS [40]. Some studies have been conducted to show the benefit of other vitamins. A 16-year follow-up study of 401 women which compared those who developed PMS and those who did not concluded that a low level of vitamin D was not associated with increased risk of PMS [41]. Existing studies on the use of vitamins as treatment for PMS are sparse, and further studies are needed to recommend it as effective treatment.

\section{Non-pharmacological treatments Herbal preparations}

The use of herbs in PMS have not been thoroughly evaluated in randomized clinical trials. Some studies have investigated the efficacy of Chinese herbals in treatment of PMS symptoms [42]. The evidence from these studies is too limited due to poor study design, and hence too poor to confirm the benefit of herbals in relieving PMS symptoms [42]. A review of Chinese herbals for PMS did not reveal any strong evidence to support their claim [43]. The herbs eval- uated in these studies include St. John's wort, gingko, peppermint, Vitex agnus-castus, analgesic root, turmeric, tangerine leaf, and bitter orange, among others [42, 43]. St. John's wort is mostly used by women with PMS, although there are insufficient data in published literature to support its use. Further extensive clinical trials are required to support the use of herbal agents.

\section{Dietary and lifestyle modification}

Dietary interventions have previously been suggested for management of PMS symptoms, but have lacked adequate research documentation. These modifications include reducing caffeine intake, and varying intake of carbohydrates or fatty foods. No particular diet has been fully demonstrated to be effective for PMS. The dietary approach for PMS should be extensively investigated. Eating less salt, more fruits and vegetables, and drinking plenty of water are recommended for women predominantly experiencing bloating, constipation, breast tenderness or swelling [44]. A study demonstrated that taking a carbohydrate-rich beverage prior to menses improved PMS symptoms [44]. Excessive alcohol intake seems to cause sleeping disorders; some women sleep either too long or too short. Nutritional guidelines should be developed for use of diet in PMS; it is cheap with little or no side effects, and puts the woman in charge of her treatment.

Exercise is another primary non-pharmacological approach to relieving PMS, and most studies have shown a benefit [45]. In these studies, exercise improved mood symptoms and physical symptoms such as breast tenderness and bloating [45]. Since regular exercise has other health benefits in addition to relief of PMS symptoms, it can be recommended as a first-line non-pharmacological treatment.

Stress, anxiety, and other psychosocial conditions could worsen PMS symptoms [46, 47]. Women living with PMS could experience difficulties in interpersonal relationships, at work or school, and with general daily living. Therefore, relaxation therapy can actually help to relieve stress and anxiety of daily life. Some randomized controlled trials demonstrated that relaxation resulted in a significant reduction in symptoms of PMS $[46,67]$. Relaxation techniques may include techniques such as meditation, muscle relaxation or self-hypnosis.

\section{Cognitive behavior therapy}

Relatively little attention has been directed towards the potential benefits of cognitive behavior therapy; however, it may be beneficial to only a few women with PMS [47]. A meta-analysis review of seven trials showed improvement in depression and functioning for women with PMS [48]. One study suggested that bright light therapy may be beneficial to some women with PMS [49]. Since the risk of cognitive behavior therapies is low, further studies are needed to support their use as first-line treatment.

\section{Conflict of interest}

The authors declare no conflict of interest. 


\section{References}

1. O’Brien PM, Bäckström T, Brown C, et al. Towards a consensus on diagnostic criteria, measurement and trial design of the premenstrual disorders: the ISPMD Montreal consensus. Arch Womens Ment Health 2011; 14: 13-21.

2. American College of Obstetricians and Gynecologists. Guidelines for Women's Health Care: A Resource Manual. $4^{\text {th }}$ ed. American College of Obstetricians and Gynecologists; Washington, DC: 2014; 607-13.

3. American Psychiatric Association. Diagnostic and Statistical Manual of Mental Disorders. $5^{\text {th }}$ ed. American Psychiatric Association; Washington, DC 2013.

4. Rapkin AJ, Winer SA. Premenstrual syndrome and premenstrual dysphoric disorder: quality of life and burden of illness. Exp Rev Pharmacoeconom Outcomes Res 2014; 9: 157-70.

5. Wittchen HU, Becker E, Lieb R, Krause P. Prevalence, incidence and stability of premenstrual dysphoric disorder in the community. Psychol Med 2002; 32: 119-32.

6. Potter J, Bouyer J, Trussell J, Moreau C. Premenstrual syndrome prevalence and fluctuation over time: results from a French population-based survey. J Womens Health (Larchmt) 2009; 18: 31-9.

7. Logue CM, Moos RH. Perimenstrual symptoms: prevalence and risk factors. Psychosom Med 1986; 48: 388-414.

8. Rapkin AJ, Lewis EI. Treatment of premenstrual dysphoric disorder. Womens Health 2013; 9: 537-56.

9. Direkvand-Moghadam A, Sayehmiri K, Delpisheh A, Kaikhavandi S. Epidemiology of premenstrual syndrome (PMS) - a systematic review and meta-analysis study. J Clin Diagn Res 2014; 8: 106-9.

10. Biggs WS, Demuth RH. Premenstrual syndrome and premenstrual dysphoric disorder. Am Fam Physician 2011; 84: 918-24.

11. Halbreich U. The etiology, biology, and evolving pathology of premenstrual syndromes. Psychoneuroendocrinology 2003; 28 (Suppl 3): 55-99.

12. Hammarbäck S, Bäckström T, Holst J, von Schoultz B, Lyrenäs S. Cyclical mood changes as in the premenstrual tension syndrome during sequential estrogen-progestagen postmenopausal replacement therapy. Acta Obstet Gynecol Scand 1985; 64: 393-7.

13. Roca CA, Schmidt PJ, Bloch $\mathrm{M}$, et al. Implications of endocrine studies in premenstrual syndrome. Psychiatr Ann 1996; 26: 576-80.

14. Kumar P, Sharma A. Gonadotropin-releasing hormone analogs: understanding advantages and limitations. J Hum Reprod Sci 2014; $7:$ 170-4.

15. Jahanfar S, Lye MS, Krishnarajah IS. The heritability of premenstrual syndrome. Twin Res Hum Genet 2011; 14: 433-6.

16. El-Rahim Elnager MA, Awed Howida Abu Ellife M. Self-care measures regarding premenstrual syndrome among female nursing students. Int J Nurs Didact 2015; 5: 1-10.

17. Abraham GE. Nutritional factors in the etiology of premenstrual tension syndromes. J Reprod Med 1983; 28: 446-64.

18. Arnold LM, Hudson JI, Keck PE, Auchenbach MB, Javaras KN, Hess EV. Comorbidity of fibromyalgia and psychiatric disorders. J Clin Psychiatry 2006; 67: 219-25.

19. Moldin SO, Scheftner WA, Rice JP, Nelson E, Knesevich MA, Akiskal H. Association between major depressive disorder and physical illness. Psychol Med 1993; 23: 755-61.

20. Gehlert S, Song IH, Chang CH, Hartlage SA. The prevalence of premenstrual dysphoric disorder in a randomly selected group of urban and rural women. Psychol Med 2009; 39: 129-36.
21. Marjoribanks J, Brown J, O’Brien PM, Wyatt K. Selective serotonin reuptake inhibitors for premenstrual syndrome. Cochrane Database Syst Rev 2013; 6: CD001396.

22. Steiner M, Steinberg S, Stewart D, et al. Fluoxetine in the treatment of premenstrual dysphoria. N Engl J Med 1995; 332: 1529-34.

23. Steiner M, Korzekwa M, Lamont J, et al. Intermittent fluoxetine dosing in the treatment of women with premenstrual dysphoria. Psychopharmacol Bull 1997; 33: 771-4.

24. Eriksson E, Hedberg MA, Andersch B, Sundblad C. The serotonin reuptake inhibitor paroxetine is superior to the noradrenaline reuptake inhibitor maprotiline in the treatment of premenstrual syndrome. Neuropsychopharmacology 1995; 12: 167-76.

25. Freeman EW, Rickerls K, Sondheimer SJ. Fluvoxamine for premenstrual dysphoric disorder: a pilot study. J Clin Psychiatry 1996; 57 (Suppl 8): 56.

26. Wikander I, Sunblad C, Andersch B, et al. Citalopram in premenstrual dysphoria: is intermittent treatment during luteal phases more effective than continuous medication throughout the menstrual cycle? J Clin Psychopharmacol 1998; 18: 390-8.

27. Freeman EW, Rickels K, Yonkers KA, Kunz NR, McPherson M, Upton GV. Venaflaxine in the treatment of premenstrual dysphoric disorder. Obstet Gynecol 2001; 98: 737-44.

28. Graham CA, Sherwin BB. A prospective treatment study of premenstrual symptoms using a triphasic oral contraceptive. J Psychosom Res 1992; 36: 257-66.

29. Freeman EW, Halbreich U, Grubb GS, et al. An overview of four studies of a continuous oral contraceptive (levonorgestrel $90 \mathrm{mcg} /$ ethinyl estradiol $20 \mathrm{mcg}$ ) on premenstrual dysphoric disorder and premenstrual syndrome. Contraception 2012; 85: 437-45.

30. Freeman EW. Evaluation of a unique oral contraceptive (Yasmin) in the management of premenstrual dysphoric disorder. Eur J Contracept Reprod Health Care 2002; 7 (Suppl 3): 27-34.

31. Freeman E, Rickels K, Sondheimer SJ, et al. Ineffectiveness of progesterone suppository treatment for premenstrual syndrome. JAMA 1990; 264: 349-53.

32. Freeman EW, Rickels K, Sondheimer SJ, et al. A double-blind trial of oral progesterone, alprazolam, and placebo in treatment of severe premenstrual syndrome. JAMA 1995; 274: 51-7.

33. Mortola JF. Applications of gonadotropin-releasing hormone analogues in the treatment of premenstrual syndrome. Clin Obstet Gynecol 1993; 36: 753-63.

34. Nevatte T, O’Brien PM, Bäckström T, et al.; Consensus Group of the International Society for Premenstrual Disorders. ISPMD consensus on the management of premenstrual disorders. Arch Womens Ment Health 2013; 16: 279-91.

35. Mortola JF, Girton L, Fischer U. Successful treatment of severe premenstrual syndrome by combined use of gonadotropin-releasing hormone agonist and estrogen/progestin. J Clin Endocrinol Metab 1991; 72: 252A-F.

36. Smith S, Rinehart JS, Ruddock VE, et al. Treatment of premenstrual syndrome with alprazolam: results of a double-blind, placebo-controlled, randomized crossover clinical trial. Obstet Gynecol 1987; 70: 37-43.

37. Brown CS, Ling FW, Farmer RG, et al.: Buspirone in the treatment of premenstrual syndrome. Drug Ther 1990; 20 (Suppl): 112-21.

38. Thys-Jacobs S, Starkey P, Bernstein D, Tian J; Premenstrual Syndrome Study Group. Calcium carbonate and the premenstrual 
syndrome: effects on premenstrual and menstrual symptoms. Am J Obstet Gynecol 1998; 179: 444-52.

39. Ghanbari Z, Haghollahi F, Shariat M, Foroshani AR, Ashrafi M. Effects of calcium supplement therapy in women with premenstrual syndrome. Taiwan J Obstet Gynecol 2009; 48: 124-9.

40. Kashanian M, Mazinani R, Jalalmanesh S. Pyridoxine (vitamin B6) therapy for premenstrual syndrome. Int J Gynaecol Obstet 2007; 96: 43-4.

41. Bertone-Johnson ER, Hankinson SE, Forger NG, et al. Plasma 25-hydroxyvitamin D and risk of premenstrual syndrome in a prospective cohort study. BMC Womens Health 2014; 14: 56.

42. Jang SH, Kim DI, Choi MS. Effects and treatment methods of acupuncture and herbal medicine for premenstrual syndrome/ premenstrual dysphoric disorder: systematic review. BMC Complement Altern Med 2014; 14: 11.

43. Jing Z, Yang X, Ismail KM, Chen X, Wu T. Chinese herbal medicine for premenstrual syndrome. Cochrane Database Syst Rev 2009; 1: CD006414.

44. Sayegh R, Schiff I, Wurtman J, et al. The effect of a carbohydrate-rich beverage on mood, appetite, and cognitive function in women with premenstrual syndrome. Obstet Gynecol 1995; 86: 520-8.

45. Prior JC, Vigna Y, Alojada N. Conditioning exercise decreases premenstrual symptoms: a prospective controlled three-month trial. Eur J Appl Physiol 1986; 55: 349-55.

46. Goodale IL, Domar AD, Benson H. Alleviation of premenstrual syndrome symptoms with the relaxation response. Obstet Gynecol 1990; 75: 649-55.

47. Morse CA, Dinnerstein L, Farrell E, et al. A comparison of hormone therapy, coping skills training, and relaxation for the relief of premenstrual syndrome. J Behav Med 1991; 14: 469.

48. Lustyk MK, Gerrish WG, Shaver S, Keys SL. Cognitive-behavioral therapy for premenstrual syndrome and premenstrual dysphoric disorder: a systematic review. Arch Womens Ment Health 2009; 12: $85-96$.

49. Lam RW, Carter D, Misri S, et al. A controlled study of light therapy in women with late luteal phase dysphoric disorder. Psychiatry Res 1999; 86: 185-92. 